\title{
Recurrent Isolation of Extremotolerant Bacteria from the Clean Room Where Phoenix Spacecraft Components Were Assembled
}

\author{
Sudeshna Ghosh,,2,* Shariff Osman,,3,* Parag Vaishampayan,, and Kasthuri Venkateswaran ${ }^{1}$
}

\begin{abstract}
The microbial burden of the Phoenix spacecraft assembly environment was assessed in a systematic manner via several cultivation-based techniques and a suite of NASA-certified, cultivation-independent biomolecule-based detection assays. Extremotolerant bacteria that could potentially survive conditions experienced en route to Mars or on the planet's surface were isolated with a series of cultivation-based assays that promoted the growth of a variety of organisms, including spore formers, mesophilic heterotrophs, anaerobes, thermophiles, psychrophiles, alkaliphiles, and bacteria resistant to UVC radiation and hydrogen peroxide exposure. Samples were collected from the clean room where Phoenix was housed at three different time points, before (1P), during (2P), and after (3P) Phoenix's presence at the facility. There was a reduction in microbial burden of most bacterial groups, including spore formers, in samples 2P and 3P. Analysis of 262 isolates from the facility demonstrated that there was also a shift in predominant cultivable bacterial populations accompanied by a reduction in diversity during $2 \mathrm{P}$ and $3 \mathrm{P}$. It is suggested that this shift was a result of increased cleaning when Phoenix was present in the assembly facility and that certain species, such as Acinetobacter johnsonii and Brevundimonas diminuta, may be better adapted to environmental conditions found during $2 \mathrm{P}$ and $3 \mathrm{P}$. In addition, problematic bacteria resistant to multiple extreme conditions, such as Bacillus pumilus, were able to survive these periods of increased cleaning. Key Words: Phoenix-Extremotolerant-Clean room-Spacecraft assembly facility. Astrobiology 10, 325-335.
\end{abstract}

\section{Introduction}

I N THE SEARCH FOR LIFE elsewhere in the Solar System, contamination of spacecraft hardware with terrestrial microorganisms is of immense concern (NRC, 2006). Introduction of hardy microorganisms to extraterrestrial environments could potentially result in the colonization of other planets (forward contamination) or result in false positives during future life-detection experiments that seek biomolecular signatures also found in terrestrial organisms (Rummel, 2001). Consequently, it is of utmost importance to monitor and control the biological burden on surfaces of spacecraft bound for planetary protection-sensitive targets by assembling hardware in certified clean room facilities that control airflow, humidity, temperature, and air particulate concentrations. These controlled conditions, coupled with periodic cleaning with chemical detergents (NASA-KSC, 1999), result in a harsh, nutrient-limited environment that is not conducive to microbial growth (La Duc et al., 2007a). However, although the stringent conditions within these facilities are effective in reducing the overall microbial load (Venkateswaran et al., 2001; La Duc et al., 2007a), they may also select for "hardy" microorganisms capable of tolerating prolonged periods of desiccation, extremes of temperature, and exposure to UV light or hydrogen peroxide (Puleo et al., 1978; La Duc et al., 2003, 2007a; Kempf et al., 2005; Newcombe et al., 2005;). A number of microbes have been isolated that can tolerate multiple environmental stresses and have been reported to survive under simulated and actual space conditions (Newcombe et al., 2005; Osman et al., 2008a). The repeated isolation of such bacteria suggests the potential for resistant microorganisms to gain access to spacecraft components and possibly survive transport to, and residence on, the surface of Mars or moons such as Europa and Titan, all of which are potential targets of future life-detection missions.

\footnotetext{
${ }^{1}$ Biotechnology and Planetary Protection Group, Jet Propulsion Laboratory, California Institute of Technology, Pasadena, California.

${ }^{2}$ Current address: Department of Civil and Environmental Engineering, University of Michigan, Ann Arbor, Michigan.

${ }^{3}$ Current address: Lawrence Berkley National Laboratory, Berkeley, California.

*Contributed equally.
} 
Reliance on a single culture-based method to monitor environments and set limits on microbial contamination is a common practice in a variety of industries and is outlined in regulations set forth by agencies and organizations such as the U.S. Environmental Protection Agency (Hussong and Madsen, 2004), U.S. Food and Drug Administration (Maturin and Peeler, 2001), American Public Health Association (Eaton et al., 2005), and United States Pharmacopeia (Farrington, 2005). Currently, NASA also utilizes a single cultivation-based assay to quantify spores and estimate overall microbial burden (NASA, 2005; NRC, 2006). However, while it may be a valid proxy for spacecraft cleanliness, the spore count assay offers only a limited phylogenetic assessment of bacterial populations that tolerate the extreme physiological conditions in clean rooms (La Duc et al., 2003). Comprehensive isolation of the hardy organisms that persist in these conditions requires the application of a breadth of distinct cultivation media, which has been shown to increase observed biological diversity in natural environments as well as in human-engineered facilities such as pharmaceutical production units (Giovannoni et al., 1988, 1990; lizuka et al., 1998; Nagarkar et al., 2001; La Duc et al., 2007a).

Although a variety of extreme-tolerant bacteria have successfully been isolated from samples collected in NASA clean rooms during non-mission periods (La Duc et al., 2007a; Osman et al., 2007; Vaishampayan et al., 2009), no data have been collected to determine whether significant shifts occur in clean room bacterial populations as a consequence of mission activities. To that end, a systematic approach was utilized to detect a broad spectrum of highly resistant bacteria throughout the spacecraft assembly process. Recurrent isolation of bacterial types common to the characteristic environmental and chemical conditions of nutrient-limited clean rooms was demonstrated, and changes in microbial incidence were correlated with maintenance practices associated with sensitive mission hardware.

\section{Materials and Methods}

\subsection{Clean room characteristics}

All samples were collected from the Kennedy Space Center (KSC) Payload Hazardous Servicing Facility (PHSF) where the Phoenix spacecraft was assembled, tested, and prepared for launch. The PHSF is a Class $100 \mathrm{~K}(<100,000$ particles $>0.5 \mu \mathrm{m} \mathrm{ft}^{-3}$ air; Fed 209E) or ISO $8(<3,520,000$ particles $>0.5 \mu \mathrm{m} \mathrm{m}^{-3}$ ), high-efficiency particulate air (HEPA) filtered clean room facility with dimensions $32.6 \mathrm{~m}$ (wide), $18.4 \mathrm{~m}$ (long), and $28.9 \mathrm{~m}$ (high). Temperature is maintained at $21.7 \pm 3.3^{\circ} \mathrm{C}$, and relative humidity is held below a maximum limit of $60 \%$. Air is volumetrically exchanged a minimum of four times per hour, with positive pressure maintained at all times. Routine maintenance is performed whether or not a spacecraft is actually present in the clean room (NASA-KSC, 1999) and includes replacement of tacky mats at entry points, frequent wiping of work surfaces, and regular cleaning of floors with clean room-certified sanitizing agents (disinfectants, alcohol, or ultrapure water). Strict protocols require that personnel entering the clean room equip themselves with clean room-certified garments, mouth and nose coverings, nitrile gloves, and footwear designed to minimize the shedding of particulates. Further- more, before entering the controlled area of the facility, individuals are subjected to a HEPA filtered air shower.

\subsection{Environmental monitoring system of the PHSF}

Temperature, relative humidity, and airborne particle concentration were continuously monitored and recorded. An environmental monitoring system (EMS) provided realtime data acquisition, and physical measurements obtained by PHSF personnel supplemented the EMS data. Surface particulate matter, nonvolatile residue, and volatile hydrocarbons were monitored via conventional methods (NASAKSC, 1999). EMS sensor sets were located in recessed purged cabinets in the west, south, and north walls of the service bay and in similar recesses in the south wall of the airlock. Each set contains a temperature sensor, a relative humidity sensor (Vaisala model 260EX), and a laser particle counter that measures airborne particle concentration (Met One model 237A).

In general, very low numbers of airborne particles within the PHSF were recorded during all three sampling events. Briefly, the number of $0.5 \mu \mathrm{m}$ particles per cubic feet rarely exceeded 18,000 during non-operation periods (1P and 3P) and 10,000 particles during Phoenix assembly (2P). After collection of the $2 \mathrm{P}$ samples on June 27,2007 , there was a spike in particle count $\left(10,000\right.$ particlesft $\left.^{-3}\right)$ observed in the early morning hours (7:30 to 8:30 AM) compared with the spike observed later in the day (10 to $11 \mathrm{AM}$ and 1:30 to 2:30 $\mathrm{PM}$ ) for the $1 \mathrm{P}$ and $3 \mathrm{P}$ sampling events. These measurements coincided with the clean room floor maintenance period. Despite the increased human activity during the assembly period (2P), very low particle counts were observed during $2 \mathrm{P}$ overall, likely due to the rigorous maintenance of the PHSF.

\subsection{Sample collection}

Several sample sets were collected from the PHSF at three time points: prior to the arrival of the Phoenix spacecraft (1P; April 25, 2007), during the assembly and testing operations of the Phoenix spacecraft prior to its launch (2P; June 27, 2007), and after removal of the spacecraft to the launch pad (3P; August 1, 2007). Samples were collected from up to 10 different locations in the facility during each sampling event. All attempts were made to sample the same physical locations within the PHSF during each sampling event; but when mission-specific activities prevented access to specific locations, alternate sampling sites were selected. Wet surface sampling of floor and wall locations was performed with Biological Sampling Kits (BiSKits, QuickSilver Analytics, Abingdon, Maryland) as previously described (Bruckner and Venkateswaran, 2007). The BiSKits consist of three components: a macrofoam sponge mounted in a plastic casing, a screw-on plastic cover, and a screw-on bottle of sample buffer. Controls specific for each BiSKit were prepared in a Class II biohood immediately prior to sampling. The manufacturer-provided buffer was brought to a volume of $30 \mathrm{ml}$ with sterile phosphate buffered saline $(\mathrm{pH}$ 7.2) then added to the macrofoam sponge component of the BiSKit. The modified buffer was recovered from the sponge by screwing the sponge casing against the BiSKit cover several times, which allowed the sample to be collected into the attached sample bottle. A $15 \mathrm{ml}$ portion of buffer was removed 
from the sample bottle and stored in $50 \mathrm{ml}$ centrifuge tubes to serve as a sample control. The remaining modified buffer was used for sample collection in the PHSF. At each sampling location, a BiSKit sponge was moistened with the modified buffer and wiped across a surface area of $1 \mathrm{~m}^{2}$. Samples were recovered from the sponges as described above. Upon collection, the sample bottles were removed from the BiSKit casings, capped, and transferred to the laboratory for processing.

\subsection{Cultivation of bacteria}

Nine different assays, as described previously (La Duc et al., 2007a), were employed to culture bacteria from BiSKit samples to grow mesophilic heterotrophic bacteria (growth at $25^{\circ} \mathrm{C}$ on R2A plates, R2A composition in grams per liter: yeast extract 0.5 , proteose peptone 0.5 , casein hydrolysate 0.5 , glucose 0.5 , soluble starch 0.5 , sodium pyruvate 0.3 , dipotassium hydrogenphosphate 0.3 , and magnesium sulfate 0.05 ), thermophiles (growth at $65^{\circ} \mathrm{C}$ on $\mathrm{R} 2 \mathrm{~A}$ plates), psychrophiles (growth at $4^{\circ} \mathrm{C}$ on R2A plates), alkali-tolerant bacteria (growth at $\mathrm{pH} \sim 11$ on $\mathrm{R} 2 \mathrm{~A}$ plates), acidophiles (growth at $\mathrm{pH} \sim 3$ on $\mathrm{R} 2 \mathrm{~A}$ plates), halophiles ( $25 \% \mathrm{NaCl}$ on R2A plates), spore formers (heat shock at $80^{\circ} \mathrm{C}$ for $15 \mathrm{~min}$ with subsequent growth at $32^{\circ} \mathrm{C}$ on TSA plates), UV- or hydrogen peroxide-resistant bacteria, and anaerobes. UVresistant bacteria were grown on R2A medium after a portion of BiSKit samples were exposed to $1,000 \mathrm{~J} / \mathrm{m}^{2}$ of UVC radiation, while hydrogen peroxide-resistant bacteria were isolated from BiSKit samples by exposing aliquots to $5 \%$ hydrogen peroxide for 1 hour before the samples were inoculated onto R2A. Anaerobes were cultured on thioglycolate medium (Pancreatic Digest of Casein $17.5 \mathrm{~g}$, Papaic Digest of Soybean Meal $2.5 \mathrm{~g}$, dextrose $10.0 \mathrm{~g}$, sodium chloride $5.0 \mathrm{~g}$, sodium thioglycolate $1.0 \mathrm{~g}$, dipotassium phosphate $2.0 \mathrm{~g}$, methylene blue $2.0 \mathrm{mg}$, agar $0.5 \mathrm{~g}$, and distilled water $1.01)$ in a GasPak anaerobic chamber. Detailed growth conditions for all cultivable bacteria have been described previously (La Duc et al., 2007a). Portions of BiSKit samples collected from each of the sampled locations within the PHSF during each of the three sampling events were plated separately and incubated or exposed under all nine of the different conditions as mentioned above. A maximum of five colonies were picked from each plate; colonies with different morphologies were preferentially sampled and stored in semisolid medium for future analysis. The number of colonies per plate for each growth condition is given in Supplementary Tables S1, S2, and S3.

\subsection{Identification of bacterial isolates}

Stored isolates were regrown on plates under appropriate conditions to obtain pure colonies. Isolates were picked and archived at $-80^{\circ} \mathrm{C}$ on Cryobeads (ProLab Diagnostics, Wirral, UK) for further processing and analysis. Individual colonies were directly analyzed by $16 \mathrm{~S}$ rRNA gene polymerase chain reaction (PCR). Primers 27F (5'-AGA GTT TGA TCC TGG CTC AG-3') and 1492R 5'-GGT TAC CTT GTT ACG ACT T- $3^{\prime}$ ) were used to PCR amplify the nearly complete $16 \mathrm{~S}$ rRNA gene (Pace et al., 1985) under the following conditions: $10 \mathrm{~min}$ at $95^{\circ} \mathrm{C}$ for cell lysis and initial denaturation followed by 35 cycles of $95^{\circ} \mathrm{C}$ denaturation for $1 \mathrm{~min} ; 55^{\circ} \mathrm{C}$ annealing for $2 \mathrm{~min}$; and $72^{\circ} \mathrm{C}$ extension for $3 \mathrm{~min}$, followed by a $10 \mathrm{~min}$ incubation at $72^{\circ} \mathrm{C}$. Occasionally, PCR was unsuccessful, in which case genomic DNA was extracted from pure cultures by suspending cells in lysis buffer $(120 \mathrm{mM}$ sodium bicarbonate and $5 \%$ sodium dodecyl sulfate at $\mathrm{pH} \sim 8$ ) followed by three freeze-thaw cycles, then incubation at $70^{\circ} \mathrm{C}$ for 1.5 hours. DNA was subsequently purified with a MO BIO genomic DNA purification kit (MO BIO Laboratories, Carlsbad, CA). PCR amplification was performed as described above.

The purified PCR amplified products were directly sequenced without cloning at Agencourt Biosciences Corporation (Beverly, MA) with the use of 27F, 907R, and 1492R primers. The phylogenetic relationships of organisms obtained during this study were determined by comparing individual $16 \mathrm{~S}$ rDNA sequences to sequences in the public database described at http://www.ncbi.nlm.nih.gov/blast. Evolutionary trees of the isolated strains were constructed via neighbor-joining, parsimony, and maximum-likelihood methods described at http://paup.csit.fsu.edu. Bootstrapping (1,000 replicates) analysis was performed to minimize sampling artifacts. Evolutionary trees were constructed with PAUP* software (Swofford, 1990) or MEGA 4.0 (Tamura et al., 2007). The GenBank accession numbers of the 262 isolates identified during this study are EU977585 to EU977841 (257 strains) and EU861362 to EU861366 (5 strains).

\subsection{Culture-independent analyses}

Adenosine triphosphate-based bioluminescence assay was used to determine the total adenosine triphosphate (ATP) and intracellular ATP as described previously (Venkateswaran et al., 2003; La Duc et al., 2007a; Fajardo-Cavazos et al., 2008). Briefly, to determine total ATP (total microbial population), $0.1 \mathrm{ml}$ sample aliquots (4 replicates) were each combined with $0.1 \mathrm{ml}$ of a cell lysing detergent (benzalkonium chloride) and then incubated at room temperature for $1 \mathrm{~min}$ prior to the addition of $0.1 \mathrm{ml}$ of luciferin-luciferase reagent. The sample was mixed, and the resulting bioluminescence was measured with a luminometer. To determine intracellular ATP (total viable microbial population), $0.1 \mathrm{ml}$ of an ATP-eliminating reagent (apyrase, adenosine deaminase) was added to a $1 \mathrm{ml}$ portion of the sample, mixed, and allowed to incubate for $30 \mathrm{~min}$ to remove any extracellular ATP, after which the assay for ATP was carried out as described above. The assay possesses a dynamic range of $5 \times 10^{-12} \mathrm{M}$ to $10^{-7} \mathrm{M}$ ATP, with one relative luminescence unit (RLU) corresponding to $\sim 2 \times 10^{-14} M$ ATP as determined by linear regression analysis of standard curves with known ATP concentrations (Venkateswaran et al., 2003). As previously established, 1 RLU is approximately equal to 1 colony-forming unit (CFU) (La Duc et al., 2007a).

\subsection{Statistical analysis}

Statistical analysis was carried out via the coin package in the R software environment (version 2.4.1). The nonparametric Kruskal-Wallis test was used to detect significant differences between total and viable ATP quantities, cultivable heterotrophic bacteria, spores, and alkali-tolerant bacteria in samples collected during $1 \mathrm{P}, 2 \mathrm{P}$, and $3 \mathrm{P}$. A local database of high-quality, full-length, 16S rRNA gene sequences corresponding to type strains recognized by the Ribosomal Database Project was constructed to assist in 
identification of isolates. Isolate sequences were queried against this database ( $>97.5 \%$ sequence similarity). Sequences found in any of the three environments $(1 \mathrm{P}, 2 \mathrm{P}$, and 3P) were compiled into a single FASTA-formatted file. Comparative analysis based on $16 \mathrm{~S}$ rRNA gene sequences was performed with the use of a JPL in-house Perl script that determined exclusive and overlapping populations between all samples and constructed a Venn diagram to illustrate the recurrent isolation of bacterial species.

\subsection{Controls and lower detection limits of assays employed}

Appropriate controls were used whenever necessary. Sterile water samples served as negative controls in all culture-based and molecular assays. Solutions of pure ATP (Sigma-Aldrich, MO) and known cells (Bacillus pumilus ATCC 7061) were serially diluted and served as a standard curve for ATP analyses. Purified DNA from B. pumilus ATCC 7061 was included in the PCR amplification protocols as a positive control. To prevent false-negative results in PCR due to the presence of inhibitory substances, a known amount of DNA was extracted from B. pumilus and spiked (1 pg per reaction mixture) as an internal standard when necessary. None of the DNA extracts in this study inhibited PCR. The lower detection limits were $1 \mathrm{CFU}\left(=3.5 \times 10^{2}\right.$ to $2.6 \times 10^{3} \mathrm{CFU} / \mathrm{m}^{2}$ ) for the cultivable plate count assay and 10 $\mathrm{RLU}\left(=3.5 \times 10^{3}\right.$ to $\left.2.6 \times 10^{4} \mathrm{CFU} / \mathrm{m}^{2}\right)$ for ATP analysis.

\subsection{Definition of terms and limitations of various assays}

Biomass levels from spacecraft assembly facility samples were assessed with a commercially available ATP detection assay. This method has been shown to have a very large dynamic range and has previously been used for describing low-biomass clean room environments (La Duc et al., 2007a). In this study, total viable microbial population was determined by exclusively intracellular ATP measurements, while the total microbial population was determined by measuring the total ATP present in a given sample. While it should be noted that both measurements could conceivably include ATP from bacterial, archaeal, and eukaryotic sources, previous culture-based and molecular-based investigations of spacecraft clean rooms have shown nonbacterial microorganisms to be negligible or absent in these environments (Moissl et al., 2008). Microscopy was deemed impractical for use in assessing microbial abundance in these samples because of the low number of microorganisms typically present $\left(\sim 10^{3}\right.$ viable cells per square meter of surface area) and the scarcity of samples ( $<10 \mathrm{ml}$ per location). Moreover, the likelihood of confounding factors particularly detrimental to low biomass samples, such as autofluorescence, false positives, nonspecific binding of stain, and false negatives resulting from insufficient penetration of stain (Moter and Gobel, 2000), undermined the value of microscopy for this type of study.

\section{Results}

\subsection{Microbial burden}

A total of 10 BiSKit samples were collected from PHSF floors before (1P) and after (3P) Phoenix was housed at the facility, while 8 PHSF floor samples were collected during
Phoenix assembly (2P). In general, most samples collected during $1 \mathrm{P}$ had a higher abundance of total bacteria as well as bacteria capable of tolerating various extreme conditions, compared with both $2 \mathrm{P}$ and $3 \mathrm{P}$ samples (Table 1). The total microbial population was in the range of $1.1 \times 10^{6}$ to $1.3 \times 10^{8}$ RLU m ${ }^{-2}$, whereas the viable microbes were 2 to 3 orders of magnitude lower. Quantities of both total and viable microbes were significantly higher $(p<0.05)$ in $1 \mathrm{P}$ samples compared with 3P samples (Table 2 ). No significant differences $(p>0.05)$ were detected in ATP counts (total or viable microbes) between $1 \mathrm{P}$ and $2 \mathrm{P}$ samples. Mean viable microbial levels were 3 and 4 times higher in PHSF samples collected prior to the arrival of spacecraft components (1P) when compared with mean viable microbial levels in $2 \mathrm{P}$ and 3P samples, respectively. This trend of microbial reduction in $2 \mathrm{P}$ and $3 \mathrm{P}$ samples compared with $1 \mathrm{P}$ samples was most evident in mesophilic heterotrophs ( $\sim 20$ times lower) and spore counts ( $\sim 165$ times lower). The overall microbial abundance was comparable between $2 \mathrm{P}$ and $3 \mathrm{P}$ samples.

Cultivable mesophilic heterotrophs were detected in 24 out of 28 samples; but, at most locations (19 samples), ATP analysis indicated that less than $10 \%$ of viable microbes were capable of growth on R2A agar (Table 1). Mesophilic heterotrophs were significantly lower $(p<0.05$; Table 2$)$ among $3 \mathrm{P}$ samples compared with $1 \mathrm{P}$ samples. Spore formers were much lower in abundance than mesophilic heterotrophs and were noticeably absent in several samples. Although spore formers were isolated from all $1 \mathrm{P}$ samples, only $25 \%$ and $50 \%$ of $2 \mathrm{P}$ and $3 \mathrm{P}$ samples, respectively, harbored them. Spore former counts from 1P samples were significantly higher $(p<0.05$; Table 2$)$ than both $2 \mathrm{P}$ and $3 \mathrm{P}$ samples. In general, spore formers comprised only $0-3 \%$ of the viable bacterial population. Alkali-tolerant bacteria were abundant among the viable populations, and comparable numbers of mesophilic heterotrophs and alkali-tolerant bacteria were frequently detected. Alkali-tolerant bacterial counts were lower $(p<0.05$; Table 2$)$ among 3P samples compared with both $1 \mathrm{P}$ and $2 \mathrm{P}$ samples. Thermophiles were exclusively detected among 1P samples. Similarly, with the exception of two samples, UV-resistant bacteria were detected primarily in 1P samples (Table 3). Psychro-tolerant bacteria were present among nine $1 \mathrm{P}$ samples and three $2 \mathrm{P}$ samples but were not isolated from the $3 \mathrm{P}$ samples. Bacteria resistant to hydrogen peroxide were rare and detected in only three 3P samples. Bacteria could not be cultivated under high salt and acidic conditions from any of the samples tested.

The percentage of various extreme-tolerant bacteria with respect to the entire viable microbial population (as measured by intracellular ATP) was calculated for all bacterial categories (Table 1). Less than $10 \%$ of the total viable microbial populations were able to grow in R2A medium under mesophilic conditions in $1 \mathrm{P}$ samples, and this percentage decreased through the following sampling events (2P and $3 \mathrm{P})$. In contrast, alkali-tolerant bacteria constituted a larger portion of the microbial population during the actual assembly of Phoenix (2P samples; $~ 8 \%$ ) compared with time periods when the clean room was not operational but was nevertheless maintained according to clean room specifications (1P and $3 \mathrm{P}$ ). Spore formers represented less than a percent of total viable microbes and less than $10 \%$ of the mesophilic heterotrophic bacteria for all the sampling events. 


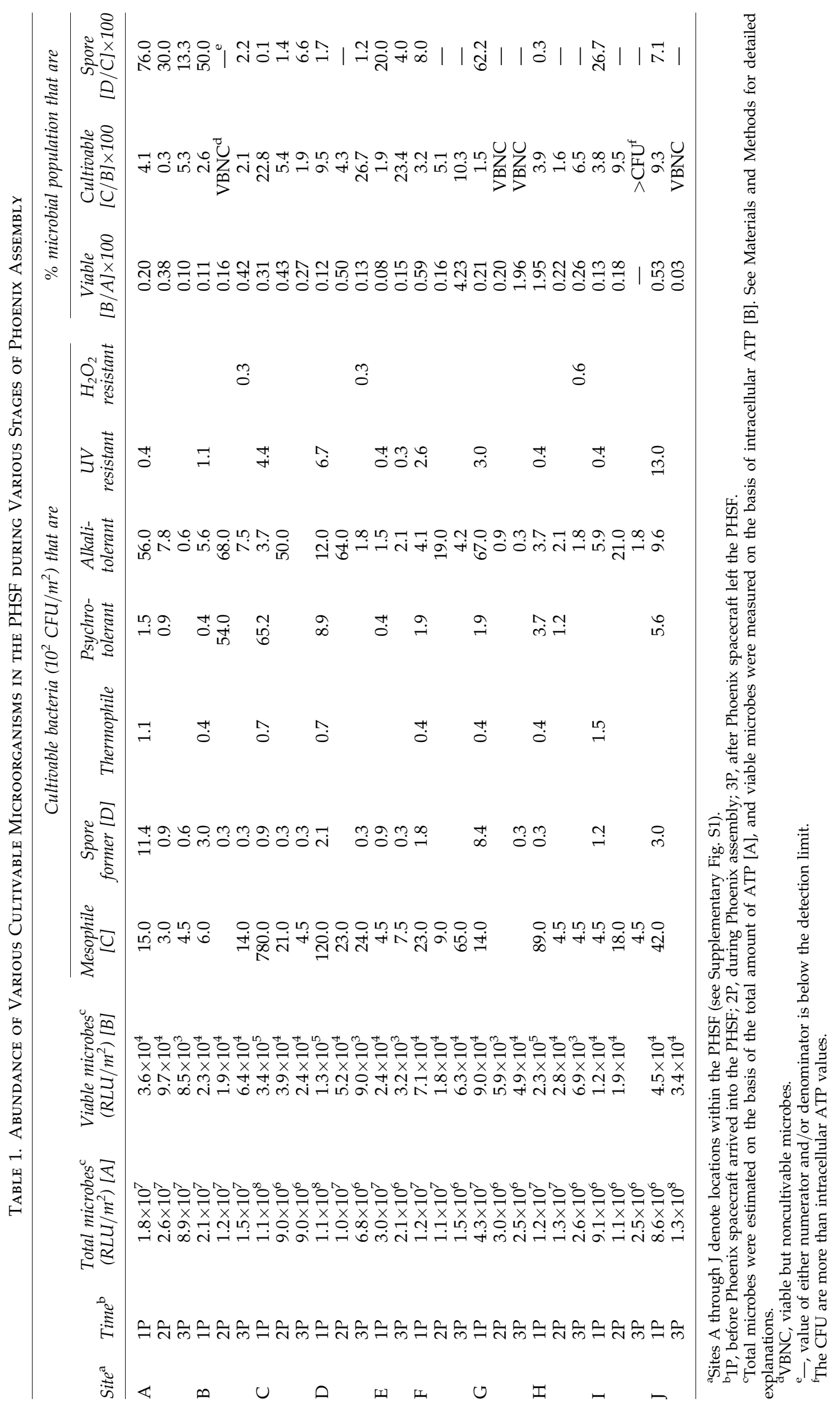


Table 2. Statistical Analysis of Microbial Abundance in the PHSF during Various Stages of Phoenix Assembly (1P Pre-Phoenix, 2P during Phoenix, 3P Post-Phoenix)

\begin{tabular}{lclll}
\hline & \multicolumn{3}{c}{ Kruskal-Wallis test $(\mathrm{p}$ values) } \\
\cline { 2 - 5 } Microbes & $1 P-2 P-3 P$ & $1 P-2 P$ & $2 P-3 P$ & $3 P-1 P$ \\
\hline Total ATP & $0.007^{*}$ & 0.088 & 0.052 & 0.021 \\
Viable ATP & 0.056 & 0.124 & 0.950 & $0.021^{*}$ \\
Mesophiles & 0.071 & 0.069 & $0.003^{*}$ & $0.044^{*}$ \\
Alkali-tolerant & $0.002^{*}$ & 0.391 & 0.577 & $0.004^{*}$ \\
Spore formers & $0.000^{*}$ & $0.000^{*}$ & $0.000^{*}$ \\
\hline
\end{tabular}

*Statistically significant based on $p<0.05$.

\subsection{Prevalence and diversity of cultivable bacteria}

Cultivable bacterial diversity of the PHSF during various stages of Phoenix assembly was assessed by $16 \mathrm{~S}$ rRNA gene sequencing. The diversity of cultivated bacteria $(n=262$ strains) and the number of novel bacteria (17 species comprising 40 strains) detected are shown in Table 3 and Fig. 1. In general, a more diverse group of bacteria was obtained from $1 \mathrm{P}$ compared with $2 \mathrm{P}$ and $3 \mathrm{P}$. Furthermore, 1P harbored a greater abundance of previously unknown bacteria. During $1 \mathrm{P}$ sampling, 32 strains belonging to 11 novel bacteria were obtained, whereas only 7 strains (6 species) from $2 \mathrm{P}$ and 1 strain from 3P samples exhibited $<97.5 \% 16 \mathrm{~S}$ rRNA gene sequence similarities with previously known species. A higher number of isolates were obtained when samples were grown under mesophilic (66 strains) and high alkaline (86 strains) conditions compared with other cultural conditions employed during this study. Strains obtained under other physiological and exposure conditions were spore formers (36 strains), psychro-tolerant bacteria (30 strains), thermophilic bacteria (8 strains), facultative anaerobes (25 strains), $\mathrm{UV}_{254}$-resistant bacteria (11 strains), and hydrogen peroxideresistant bacteria (5 strains).

A distinct shift in bacterial populations was observed from $1 \mathrm{P}$ to $2 \mathrm{P}$ and $3 \mathrm{P}$ samples. Isolates collected from the prePhoenix period (1P) included representatives from the Proteobacteria (9 species), low-GC Gram-positive organisms (Firmicutes, 17 species), high-GC Gram-positive organisms (Actinobacteria, 17 species), and one each of Bacteroidetes and Deinococcus groups. Strains collected during Phoenix (2P) belonged to members of the Proteobacteria, Firmicutes, and Actinobacteria, while only Proteobacteria and Firmicutes members were detected from post-Phoenix samples (3P). The markedly reduced diversity during $2 \mathrm{P}$ and $3 \mathrm{P}$ was accompanied by increased prevalence of Acinetobacter johnsonii and Brevundimonas diminuta (Table 3). Analysis of overlapping cultivable bacterial populations from all 3 sampling events (Fig. 2) revealed that 31 of the 43 species present during $1 \mathrm{P}$ were no longer detected during $2 \mathrm{P}$ and $3 \mathrm{P}$. Only 2 species, $B$. pumilus and B. diminuta, were detected during all 3 sampling events.

\subsection{Functional diversity}

When samples were grown under mesophilic conditions, a total of 21 species was isolated, 5 of which were novel species. Only 4 were found in more than one sampling event (Table 3). Characterization of alkali-tolerant and psychro- tolerant strains further demonstrated physiological diversity present in the PHSF. Direct cultivation in high-alkaline medium yielded a total of 23 species, 2 of which were novel; low-temperature conditions resulted in the isolation of 18 species, 6 of which were novel. Acinetobacter johnsonii and $B$. diminuta dominated the mesophilic and alkali-tolerant bacteria recovered from $2 \mathrm{P}$ and $3 \mathrm{P}$, while a much more diverse group of organisms were isolated from 1P samples. Psychro-tolerant bacteria predominantly belonged to Proteobacteria and Actinobacteria, with no single species dominating the isolates (Table 3 ). Of the 6 UV-resistant bacterial species analyzed, 4 belonged to Firmicutes. All 25 strains isolated under anaerobic conditions were also able to grow aerobically.

Spore formers demonstrated diverse metabolic capabilities: 11 species were detected from the spore-favoring heat shock assay, 7 were obtained from high-alkaline media, 7 were cultured under anaerobic conditions, 5 were isolated on neutral pH R2A under mesophilic conditions, 2 were isolated under psychro-tolerant conditions, 4 survived high UV exposure, and 1 was determined to be both hydrogen peroxide resistant and thermophilic. Although species of Bacillus were found under several of the growth/exposure conditions employed, they were not isolated when samples were exposed to low $\mathrm{pH}$ or high-salinity growth conditions. It should be noted that a single species, B. pumilus, was found to tolerate nearly all experimental stresses, including growth at high temperature $\left(\sim 65^{\circ} \mathrm{C}\right)$ and exposure to hydrogen peroxide conditions, but was not isolated under lowtemperature conditions $\left(4-8^{\circ} \mathrm{C}\right)$. Eight thermophilic bacterial strains were obtained from the $1 \mathrm{P}$ samples; in addition to a single isolate of B. pumilus, 7 strains of Geobacillus stearothermophilus were collected.

\subsection{Novel bacterial diversity}

The phylogenetic affiliations of novel bacteria isolated in this study are depicted in Fig. 1. Among the 17 species deemed novel, based on 16S rRNA gene sequence analysis (Stackebrandt and Goebel, 1994), there were 6 mesophilic heterotrophs, 6 psychro-tolerant, 5 spore-forming, and 3 alkali-tolerant bacteria not as yet described in the literature. Several of these novel strains exhibited tolerance to multiple physiological conditions. For example, some of the psychroand alkali-tolerant strains also formed spores (Table 3). Strains isolated as UV-resistant or thermophilic belonged to already-described species. Except for one each of Bacillus and Georgenia species, novel species were not found in more than 
Table 3. Bacteria That Could Grow at Various Physiological Conditions

\begin{tabular}{|c|c|c|c|c|c|c|c|c|c|c|c|c|c|}
\hline \multirow[b]{3}{*}{ Phyla/Species } & \multicolumn{13}{|c|}{ \# of strains isolated under the conditions that promotes the growth/conditons of } \\
\hline & \multicolumn{3}{|c|}{ Mesophilic heterotrophs } & \multicolumn{3}{|c|}{ Alkali-tolerant } & \multicolumn{2}{|c|}{ Psychro-tolerant } & \multicolumn{3}{|c|}{ UV resistant } & \multicolumn{2}{|c|}{ Aero-tolerant } \\
\hline & $1 P$ & $2 P$ & $3 P$ & $1 P$ & $2 P$ & $3 P$ & $1 P$ & $2 P$ & $1 P$ & $2 P$ & $3 P$ & $1 P$ & $2 P$ \\
\hline \multicolumn{14}{|l|}{ Firmicutes } \\
\hline Bacillus flexus & 1 & 1 & & & & & & & 3 & & & & \\
\hline Bacillus humi & & & & & & & & & 1 & & & & \\
\hline Bacillus odysseyi & & & & & & & & & & & & & \\
\hline Bacillus oshimensis & & & & 2 & & & & & & & & & \\
\hline Bacillus pseudalcaliphilus & & & & 1 & & & & & & & & & \\
\hline Bacillus pumilus & 1 & 1 & & 1 & 1 & 2 & & & 1 & & 1 & & 1 \\
\hline Bacillus thuringiensis & 2 & & & 4 & 1 & & & & & & & 1 & 2 \\
\hline Brevibacillus invocatus & & & & & & & 1 & & & & & 1 & \\
\hline Enterococcus faecium & & & & & & & & & & & & 2 & \\
\hline \multicolumn{14}{|l|}{ Geobacillus stearothermophilus } \\
\hline Oceanobacillus iheyensis & & & & 1 & & & & & & & & & \\
\hline Oceanobacillus profundus & & & & 1 & & & & & & & & & \\
\hline Paenibacillus chitinolyticus & & & & & & & & & & & & & \\
\hline Paenibacillus jamilae & & & & & & & & & & & & 2 & \\
\hline Paenibacillus lactis & & & & & & & & & & & & & \\
\hline Paenibacillus xylanilyticus & 1 & & & & & & & & 2 & & & & \\
\hline Staphylococcus epidermidis & & & & & & & & & & & & & 4 \\
\hline Bacillus sp. & 1 & & & 3 & 1 & & 1 & & & & & 1 & \\
\hline Paenibacillus sp. & & & & & & & & & & & & & \\
\hline Proteobacteria & & & & & & & & & & & & & \\
\hline Acinetobacter johnsonii & & 6 & 8 & & 13 & 7 & & 2 & & & & & \\
\hline Brevundimonas diminuta & & 7 & 15 & 1 & 11 & 15 & & & & & & & \\
\hline Chelatococcus asaccharovorans & 1 & & & & & & & & & & & & \\
\hline Methylobacterium aquaticum & 1 & & & & & & & & & & & & \\
\hline Pseudomonas oryzihabitans & 6 & & & & & & 5 & & & & & 4 & \\
\hline Sphingomonas dokdonensis & & & & & 1 & & & & & & & & \\
\hline Sphingomonas yunnanensis & 2 & & & & & & 1 & & & & & & \\
\hline Stenotrophomonas rhizophila & & & & & & 1 & & & & & & & \\
\hline Variovorax paradoxus & & & & & & & 1 & & & & & & \\
\hline Herbaspirillum sp. & & & & & & & 3 & & & & & & \\
\hline Mesorhizobium sp. & 1 & & & & & & & & & & & & \\
\hline Methylobacterium sp. & & 1 & & & & & & & & & & & \\
\hline Serratia sp. & & & & & & & & & & & & & 1 \\
\hline Sphingomonas sp. & & & & & & & 1 & & & & & & \\
\hline Actinobacteria & & & & & & & & & & & & & \\
\hline Agrococcus jenensis & & & & 1 & & & & & & & & & \\
\hline Arthrobacter polychromogenes & & & & & & & 1 & 2 & & & & & \\
\hline Blastococcus aggregatus & & & & & & & & & & & & 1 & 1 \\
\hline Cellulosimicrobium funkei & & & & 2 & & & & & & & & & \\
\hline Curtobacterium luteum & & & & & & & & 1 & & & & 1 & 2 \\
\hline Dietzia maris & 1 & & & 1 & & & 3 & & & & & & \\
\hline Janibacter terrae & & & & 1 & & & & & & & & & \\
\hline Kineococcus marinus & 1 & & & & & & & & & & & & \\
\hline Kocuria rosea & & & & & 2 & & & & & & & & \\
\hline Labedella kawkjii & & & & & & & & 1 & & & & & \\
\hline Microbacterium arborescens & & & & & 1 & & & & & & & & \\
\hline Microbacterium testaceum & 2 & & & 3 & 1 & & & & 2 & & & & \\
\hline Mycobacterium houstonense & & & & & & & & & & 1 & & & \\
\hline Nocardioides oleivorans & 1 & & & 1 & & & & & & & & & \\
\hline Plantibacter flavus & & & & & 2 & & & 1 & & & & & \\
\hline Rhodococcus fascians & & & & & & & 2 & & & & & & \\
\hline Rhodococcus globerulus & & & & 1 & & & & & & & & & \\
\hline Rhodococcus kroppenstedtii & & & & & 1 & & & & & & & & \\
\hline Sanguibacter inulinus & & 1 & & & & & & & & & & & \\
\hline Arthrobacter sp. & 1 & & & & & & & & & & & & \\
\hline Blastococcus sp. & & & & & & & 1 & & & & & & \\
\hline Georgenia sp. & & & & 2 & & & & & & & & & 1 \\
\hline Kineosporia sp. & 2 & & & & & & & & & & & & \\
\hline Sanguibacter sp. & & & & & & & & 1 & & & & & \\
\hline Rhodococcus sp. & & & & & & & 1 & & & & & & \\
\hline Others & & & & & & & & & & & & & \\
\hline Spirosoma aquatica & & & & & & & 1 & & & & & & \\
\hline Deinococcus sp. & 1 & & & & & & & & & & & & \\
\hline
\end{tabular}




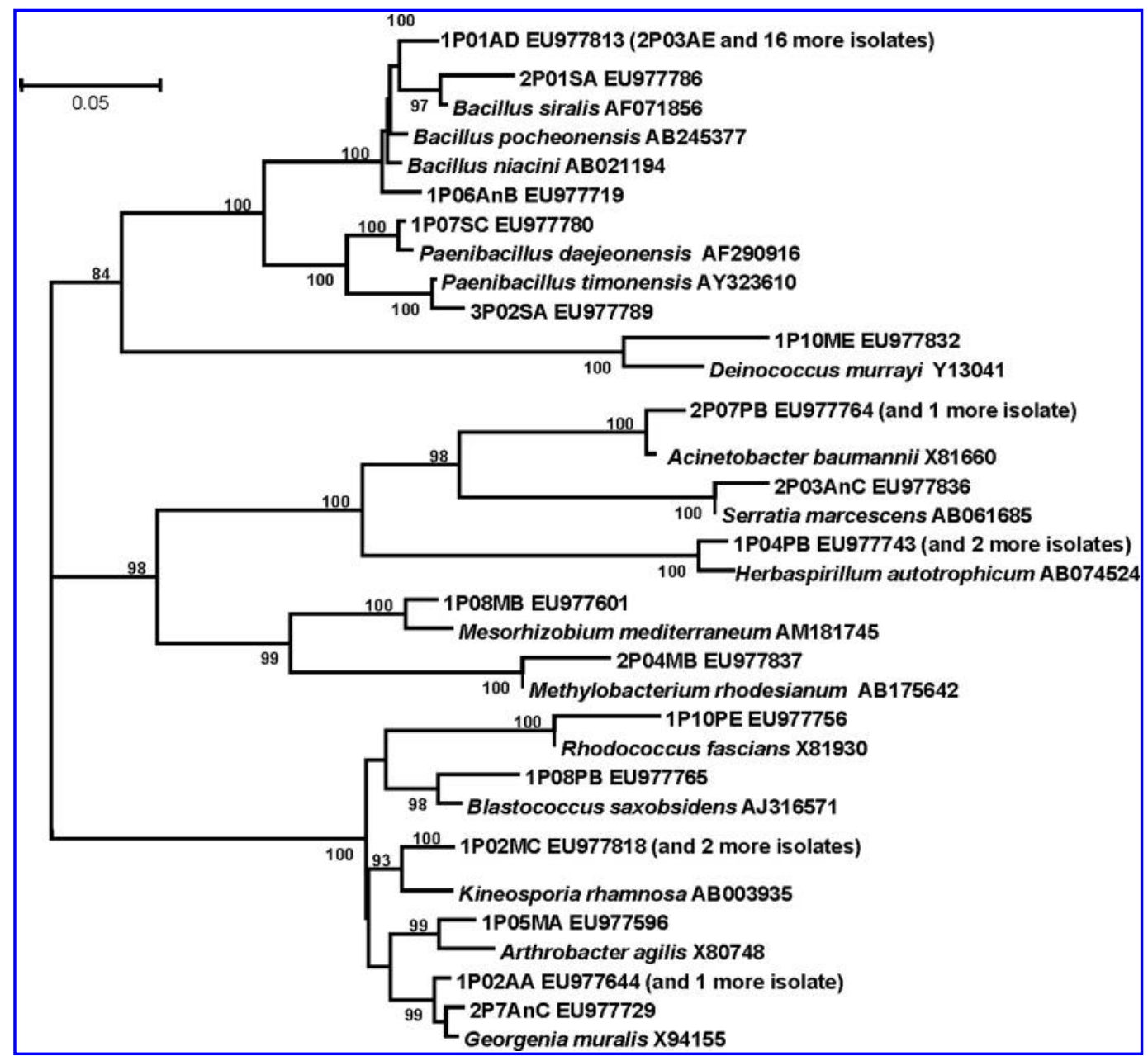

FIG. 1. Phylogenetic tree (maximum parsimony) of cultivable bacteria isolated from the PHSF. GenBank accession numbers for 16S rRNA gene sequences of the novel isolates are shown following the strain number designations. Where the novel strains were isolated more than once, the frequency numbers are given in parenthesis. Only novel strains identified within this study showing $<97.5 \%$ similarities (16S rRNA gene) to the closest described species are included. Branching percentage values were determined with the use of 1000 bootstraps. The scale bar shows a $5 \%$ estimated difference in nucleotide sequence positions.

a single sampling event; however, several isolates of the same novel species were often found in various locations of that sampling event. One novel alkali-tolerant species identified as B. horneckiae was found in at least 6 locations during the $1 \mathrm{P}$ sampling event and has recently been described (Vaishampayan et al., 2009) by way of a variety of metabolic and phylogenetic analyses. Further research is under way to describe other bacterial species isolated during this study.

\section{Discussion}

The ratios between total ATP content, intracellular ATP content, and CFU counts are reliable indicators of relative microbial abundances on clean room surfaces (Venkateswaran et al., 2003; La Duc et al., 2007a). Ratios of intracellular ATP content to total ATP were very low (0.03-4.3\%) in all samples irrespective of sampling events, which is consistent with surface samples obtained from a number of certified clean rooms ranging from class 10 to 100K (La Duc et al., 2007a). As expected, cultivable bacteria accounted for a minor portion of the total viable bacteria in most samples, with very low ratios of CFU to viable microbes $(<10 \%)$ for the majority of samples. The challenging environmental conditions in the PHSF clean room made the ATP assay used in this study particularly useful for assessing bacterial contamination. Approximately $15 \%$ of collected samples yielded no cultivable mesophilic heterotrophs, but the samples possessed intracellular ATP values well above the assay's detection limit (Table 1). Previous molecular microbial community analyses of samples devoid of mesophilic heterotrophs have confirmed the presence of a number of other bacteria that either have never been cultured or require very specific culture conditions (Venkateswaran et al., 2003; La Duc et al., 2007a). Although the extremely low cellular biomass levels found in clean rooms makes DNA extraction and PCR amplification from individual samples unreliable, pooled samples from each of the sampling events discussed herein have revealed the presence of these difficult-to-culture organisms (Vaishampayan et al., unpublished data). A single sample yielded mesophilic heterotrophs despite having intracellular ATP measurement below detection limit (Table 1). Such outliers demonstrate that the distribution of microbes 


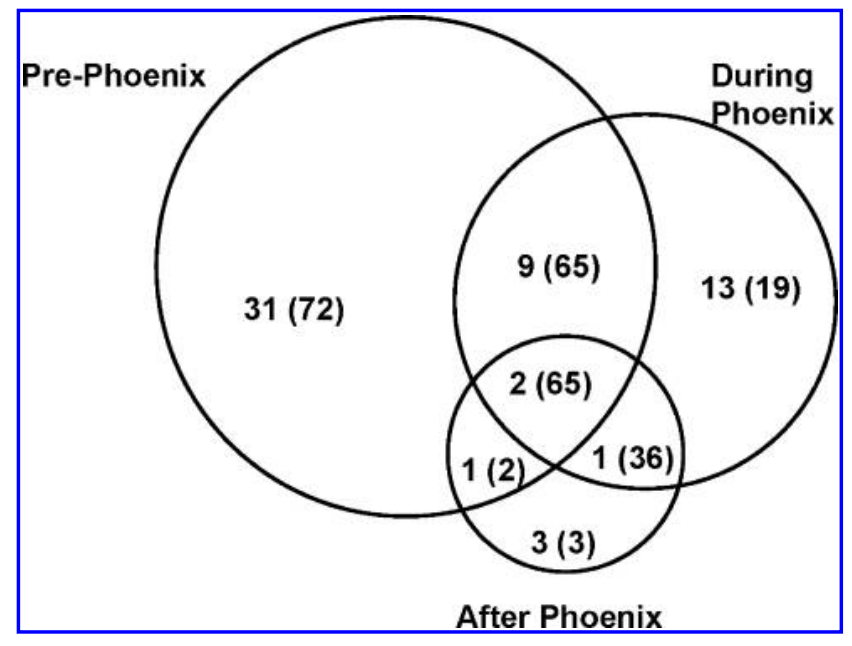

FIG. 2. Recurrent isolation of bacterial species during the maintenance of the PHSF for the Phoenix mission. Schematic drawing shows the detected bacterial species found in various sampling events of the PHSF clean room. Overlaps between the sampling events are indicated by the arrangement of the circles. The number of bacterial species found exclusive or overlapping with the environments is shown individually, and the value in parentheses denotes the number of strains belonging to that circle or overlap.

in any given sampling location is far from uniform and underscores the challenge of drawing statistical conclusions from low biomass samples (Venkateswaran et al., 2003).

While it has been observed that bacteria sometimes enter the clean room with humans (Puleo et al., 1978; Castro et al., 2004), other bacteria may be well adapted to the oligotrophic conditions of these environments as well as the chemicals used for cleaning the facility (La Duc et al., 2004). Furthermore, differences observed in microbial diversity and abundance between the three sampling dates suggest that these populations are not stable and may fluctuate significantly throughout the spacecraft assembly process. Compared with measurements taken before Phoenix's arrival at the PHSF (1P), samples collected during Phoenix assembly (2P) and post-Phoenix assembly (3P) displayed reduced numbers of cultivable mesophilic heterotrophs and extremophiles as well as a reduction in diversity. There was also a distinct shift in the type of bacteria isolated in the PHSF across the three sampling events. The most commonly isolated organisms from $1 \mathrm{P}$ were $B$. flexus (10 isolates) and $P$. oryzihabitans (15 isolates), while $A$. johnsonii and $B$. diminuta were most common among $2 \mathrm{P}$ and $3 \mathrm{P}$ isolates. The reduced abundance (Table 1 ) and diversity of bacteria in the $2 \mathrm{P}$ samples (Table 3 ), despite increased human activity and consistent environmental controls, strongly suggest that more stringent cleaning protocols during the presence of the Phoenix spacecraft were responsible for the shift in bacterial populations.

Previous studies have reported species of the extremely hardy, spore-forming Bacillus genus to be the most highly represented organisms ( $>85 \%$ of the cultivable population) in samples collected from spacecraft assembly facilities (Favero, 1971; Puleo et al., 1977). However, these findings were heavily influenced heat-shock procedures that optimized the detection of spores and excluded vegetative cells. In this study, cultivable bacteria from the PHSF belonged to various groups of bacteria that included spore formers as well as several non-spore-forming extremophilic species. Previous research studying several NASA clean rooms has also indicated similar bacterial profiles (La Duc et al., 2007a). While NASA planetary protection concerns about extremophilesparticularly with respect to vacuum, UV radiation, and ionizing radiation survival-have focused primarily on spore formers, the results of this study demonstrate that clean rooms host a variety of non-spore-forming bacteria that can survive extreme conditions and warrant further research into their ability to tolerate other conditions likely to be encountered during space missions.

In an attempt to develop tools that could accurately assess the risk of forward contamination, the recurrence of problematic bacteria under various culturing conditions and across multiple sampling events was compared via JPL inhouse computational modeling. This interactive database, which cataloged 16S rRNA gene sequences from all microorganisms detected during this study, revealed the prevalence of recurring microorganisms within the PHSF and allowed inferences to be made regarding the proportions of dominant taxa present in microbial populations. For example, the 1P samples showed the highest diversity of cultivable bacteria, exhibiting 43 species with 22 singletons (bacteria occurring only once; Fig. 2) (Schloss and Handelsman, 2005). However, when the $16 \mathrm{~S}$ rRNA gene sequences from the 262 isolate collection were examined as a group, 25\% $(n=65)$ of the bacterial isolates belonged to only 2 species: $B$. pumilus and $B$. diminuta.

In addition to its high occurrence, the spore-forming $B$. pumilus showed the highest versatility among the isolates, growing under 6 different culturing conditions and tolerating exposure to UV, hydrogen peroxide, and temperatures up to $65^{\circ} \mathrm{C}$ (Table 3). The isolation of B. pumilus during all three sampling periods indicates that this species was able to survive increased cleaning procedures employed while the Phoenix spacecraft occupied the PHSF, and previous research has demonstrated high tolerance of B. pumilus to extreme conditions (Kempf et al., 2005; Newcombe et al., 2005). Although the presence of B. pumilus has been noted in previous investigations of NASA clean rooms (Kempf et al., 2005; Newcombe et al., 2005; Satomi et al., 2006), the results of this investigation demonstrate its resilience to several environmental stressors that are relevant to space research. Similarly, B. diminuta was detected in all three sampling events (Fig. 2). But despite frequent detection in these samples and occurrence in other oligotrophic environments (Penna et al., 2002; Mazzola et al., 2006; Osman et al., 2008a, 2008b), the significance of $B$. diminuta in the microbial ecology of the clean room environment (La Duc and others, 2007b) remains to be elucidated.

The fluctuation of clean room contaminants over the course of spacecraft assembly has great significance for planetary protection objectives. Development of appropriate cleaning and sterilization protocols will require a solid understanding of the physiological capabilities of clean room microorganisms. Meaningful analysis of data from upcoming life-detection missions will likewise require knowledge of how these microbes are distributed within clean rooms, across assembly facilities, and on spacecraft hardware. 
Database applications capable of tracking recurrent organisms and the extent to which they can survive extreme growth/ exposure conditions will be essential for developing protocols that promote the biological integrity of NASA missions. Beyond NASA mission objectives, however, the baseline bacterial inventory/databases created during this study will have application in medical, pharmaceutical, and healthcare industrial environments where clean rooms are used.

\section{Acknowledgments}

Part of the research described in this publication was carried out at the Jet Propulsion Laboratory, California Institute of Technology, under a contract with the National Aeronautics and Space Administration. This research was funded by NASA Research Announcement (NRA) ROSES 2006 awarded to Kasthuri Venkateswaran. We are grateful to members of the JPL Biotechnology and Planetary Protection Group for technical assistance. We also appreciate the help rendered by R. Sumner, B. Petsos, Y. Salinas, and D. Vaughn during sampling. We are thankful to J. Spry, C. Conley, and J. Rummel for valuable advice and encouragement.

\section{Abbreviations}

ATP, adenosine triphosphate; CFU, colony-forming unit; EMS, environmental monitoring system; HEPA, highefficiency particulate air; KSC, Kennedy Space Center; PCR, polymerase chain reaction; PHSF, Payload Hazardous Servicing Facility; RLU, relative luminescence unit.

\section{References}

Bruckner, J.C. and Venkateswaran, K. (2007) Overview of methodologies to sample and assess microbial burden in low biomass environments. Japanese Journal of Food Microbiology 24:61-70.

Castro, V.A., Thrasher, A.N., Healy, M., Ott, C.M., and Pierson, D.L. (2004) Microbial characterization during the early habitation of the International Space Station. Microb. Ecol. 47:119 126.

Eaton, A.D., Clesceri, L.S., Rice, E.W., and Greenberg, A.E., editors. (2005) Standard Methods for the Examination of Water and Wastewater, American Public Health Association, Water Environment Federation, and American Water Works Association, Denver, CO.

Fajardo-Cavazos, P., Schuerger, A.C., and Nicholson, W.L. (2008) Persistence of biomarker ATP and ATP-generating capability in bacterial cells and spores contaminating spacecraft materials under earth conditions and in a simulated martian environment. Appl. Environ. Microbiol. 74:5159-5167.

Farrington, J.K. (2005) Environmental monitoring in pharmaceutical manufacturing-a product risk issue. American Pharmaceutical Review 8:26-30.

Favero, M.S. (1971) Microbiologic assay of space hardware. Environ. Biol. Med. 1:27-36.

Giovannoni, S.J., Delong, E.F., Olsen, G.J., and Pace, N.R. (1988) Phylogenetic group-specific oligodeoxynucleotide probes for identification of single microbial-cells. I. Bacteriol. 170:720726.

Giovannoni, S.J., Britschgi, T.B., Moyer, C.L., and Field, K.G. (1990) Genetic diversity in Sargasso Sea bacterioplankton. Nature 345:60-63.
Hussong, D. and Madsen, R.E. (2004) Analysis of environmental microbiology data from cleanroom samples. Pharmaceutical Technology, Aseptic Processing Issue, pp 10-15.

Iizuka, T., Yamanaka, S., Nishiyama, T., and Hiraishi, A. (1998) Isolation and phylogenetic analysis of aerobic copiotrophic ultramicrobacteria from urban soil. J. Gen. Appl. Microbiol. 44:75-84.

Kempf, M.J., Chen, F., Kern, R., and Venkateswaran, K. (2005) Recurrent isolation of hydrogen peroxide-resistant spores of Bacillus pumilus from a spacecraft assembly facility. Astrobiology 5:391-405.

La Duc, M.T., Nicholson, W., Kern, R., and Venkateswaran, K. (2003) Microbial characterization of the Mars Odyssey spacecraft and its encapsulation facility. Environ. Microbiol. 5:977-985.

La Duc, M.T., Kern, R.G., and Venkateswaran, K. (2004) Microbial monitoring of spacecraft and associated environments. Microb. Ecol. 47:150-158.

La Duc, M.T., Dekas, A.E., Osman, S., Moissl, C., Newcombe, D., and Venkateswaran, K. (2007a) Isolation and characterization of bacteria capable of tolerating the extreme conditions of cleanroom environments. Appl. Environ. Microbiol. 73:2600-2611.

La Duc, M.T., Stucker, T., and Venkateswaran, K. (2007b) Molecular bacterial diversity and bioburden of commercial airliner cabin air. Can. J. Microbiol. 53:1259-1271.

Maturin, L. and Peeler, J.T. (2001) Aerobic plate count. In Bacteriological Analytical Manual, U.S. Food and Drug Administration, Silver Spring, MD, chapter 3.

Mazzola, P.G., Martins, A.M., and Penna, T.C. (2006) Chemical resistance of the Gram-negative bacteria to different sanitizers in a water purification system. BMC Infect. Dis. 6:131.

Moissl, C., Bruckner, J., and Venkateswaran, K. (2008) Archaeal diversity analysis of spacecraft assembly facilities. ISME J. 2:115-119.

Moter, A. and Gobel, U.B. (2000) Fluorescence in situ hybridization (FISH) for direct visualization of microorganisms. $\underline{\text {. }}$ Microbiol. Methods 41:85-112.

Nagarkar, P.P., Ravetkar, S.D., and Watve, M.G. (2001) Oligophilic bacteria as tools to monitor aseptic pharmaceutical production units. Appl. Environ. Microbiol. 67:1371-1374.

NASA. (2005) Planetary protection provisions for robotic extraterrestrial missions, NPR 8020.12C, April 2005, Science Mission Directorate, National Aeronautics and Space Administration, Washington DC.

NASA-KSC. (1999) Launch Site Requirement Planning Group, facilities handbook of Payload Hazardous Servicing Facility (PHSF), Kennedy Space Center, Cape Canaveral, FL.

Newcombe, D.A., Schuerger, A.C., Benardini, J.N., Dickinson, D., Tanner, R., and Venkateswaran, K. (2005) Survival of spacecraft-associated microorganisms under simulated martian UV irradiation. Appl. Environ. Microbiol. 71:8147-8156.

NRC. (2006) Preventing the Forward Contamination of Mars, Committee on Preventing the Forward Contamination of Mars, National Research Council, National Academies Press, Washington DC.

Osman, S., Moissl, C., Hosoya, N., Briegel, A., Mayilraj, S., Satomi, M., and Venkateswaran, K. (2007) Tetrasphaera remsis sp. nov., isolated from the Regenerative Enclosed Life Support Module Simulator (REMS) air system. Int. J. Syst. Evol. Microbiol. 57:2749-2753.

Osman, S., Peeters, Z., LaDuc, M.T., Mancinelli, R., Ehrenfreund, P., and Venkateswaran, K. (2008a) Effect of shadowing on the survival of bacteria to conditions simulating martian atmosphere and UV-radiation. Appl. Environ. Microbiol. 74:959-970. 
Osman, S., LaDuc, M.T., Dekas, A., Newcombe, D., and Venkateswaran, K. (2008b) Microbial bioburden and diversity of commercial airline cabin air during short- and long-duration of travel. ISME J. 2:482-497.

Pace, N.R., Stahl, D.A., Lane, D.J., and Olsen, G.J. (1985) The analysis of natural microbial communities by ribosomal RNA sequences. Microb. Ecol. 9:1-56.

Penna, V.T., Martins, S.A., and Mazzola, P.G. (2002) Identification of bacteria in drinking and purified water during the monitoring of a typical water purification system. BMC Public Health 2, doi:10.1186/1471-2458-2-13.

Puleo, J.R., Fields, N.D., Bergstrom, S.L., Oxborrow, G.S., Stabekis, P.D., and Koukol, R. (1977) Microbiological profiles of the Viking spacecraft. Appl. Environ. Microbiol. 33: 379-384.

Puleo, J.R., Bergstrom, S.L., Peeler, J.T., and Oxborrow, G.S. (1978) Thermal resistance of naturally occurring airborne bacterial spores. Appl. Environ. Microbiol. 36:473-479.

Rummel, J.D. (2001) Planetary exploration in the time of astrobiology: protecting against biological contamination. $\underline{\text { Proc. }}$ Natl. Acad. Sci. U.S.A. 98:2128-2131.

Satomi, M., La Duc, M.T., and Venkateswaran, K. (2006) Bacillus safensis sp. nov., isolated from spacecraft and assembly-facility surfaces. Int. J. Syst. Evol. Microbiol. 56:1735-1740.

Schloss, P.D. and Handelsman, J. (2005) Introducing DOTUR, a computer program for defining operational taxonomic units and estimating species richness. Appl. Environ. Microbiol. 71:1501-1506.

Stackebrandt, E. and Goebel, B.M. (1994) Taxonomic note: a place for DNA-RNA reassociation and 16S RNA sequence analysis in the present species definition of bacteriology. Int. J. Syst. Bacteriol. 44:846-849.
Swofford, D. (1990) PAUP: phylogenetic analysis using parsimony, Version 2.0. Computer program distributed by the Illinois Natural Survey, Champaign, IL.

Tamura, K., Dudley, J., Nei, M., and Kumar, S. (2007) MEGA4: Molecular Evolutionary Genetics Analysis (MEGA) software version 4.0. Mol. Biol. Evol. 24:1596-1599.

Vaishampayan, P., Probst, A., Krishnamurthi, S., Ghosh, S., Osman, S., McDowall, A., Rukmani, A., Mayilraj, S., and Venkateswaran, K. (2010) Bacillus horneckiae sp. nov., isolated from a clean room where the Phoenix spacecraft was assembled. Int. J. Syst. Evol. Microbiol., doi: 10.1099/ijs.0.008979-0.

Venkateswaran, K., Satomi, M., Chung, S., Kern, R., Koukol, R., Basic, C., and White, D.C. (2001) Molecular microbial diversity of a spacecraft assembly facility. Syst. Appl. Microbiol. 24:311-320. Venkateswaran, K., Hattori, N., La Duc, M.T., and Kern, R. (2003) ATP as a biomarker of viable microorganisms in cleanroom facilities. I. Microbiol. Methods 52:367-377.

Address correspondence to: Kasthuri Venkateswaran Biotechnology and Planetary Protection Group $M / S 89$

Jet Propulsion Laboratory California Institute of Technology 4800 Oak Grove Dr. Pasadena, CA 91109

E-mail: kjvenkat@jpl.nasa.gov

Submitted 5 June 2009 Accepted 6 February 2010 

This article has been cited by:

1. Parag Vaishampayan, Shariff Osman, Gary Andersen, Kasthuri Venkateswaran . 2010. High-Density 16S Microarray and Clone Library-Based Microbial Community Composition of the Phoenix Spacecraft Assembly Clean RoomHigh-Density 16S Microarray and Clone Library-Based Microbial Community Composition of the Phoenix Spacecraft Assembly Clean Room. Astrobiology 10:5, 499-508. [Abstract] [Full Text] [PDF] [PDF Plus] [Supplementary material] 